\title{
Analysis of the evolution of gene expression patterns in flowering plants
}

\author{
Penin A.A. ${ }^{1,2 *}$, Kasianov A.S. ${ }^{3}$, Klepikova A.V. ${ }^{1}$, Gerasimov E.S. ${ }^{1,2}$, \\ Logacheva M.D. ${ }^{1,4}$ \\ ${ }^{1}$ Institute for Information Transmission Problems, RAS, Moscow, Russia \\ ${ }^{2}$ Lomonosov Moscow State University, Moscow, Russia \\ ${ }^{3}$ Vavilov Institute of General Genetics, RAS, Moscow, Russia \\ ${ }^{4}$ Skolkovo Institute of Science and Technology, Moscow, Russia \\ *e-mail: alekseypenin@gmail.com
}

Polyploidization with subsequent functional divergence (neo- and subfunctionalization) or loss of duplicated genes is one of the major mechanisms of plant genome evolution. In particular, whole genome duplications are associated with the emergence of such large clades as monocots and core eudicots. However, the patterns of these processes remain unclear in many aspects. The way to gain insight into them is the study of the evolution of gene expression profiles as soon as the expression reflects the function. Using the method of gradient busting we performed comparison of detailed expression maps for five plants, representing large groups of angiosperms: rosids (the model object Arabidopsis thaliana), asterids (Solanum lycopersicum), caryophyllids (Fagopyrum esculentum), monocots (Zea mays) and basal angiosperm Amborella trichopoda. This allowed identification of groups of genes with conserved expression profiles, present in all five species. These profiles are presumably inherited from a common ancestor of angiosperms and can be called the core pan-transcriptome of flowering plants. Based on these data it is possible to trace the genes that changed their function after duplication and ones that underwent neo- and subfunctionalization. Also, the analysis of the coexpressed groups of non-homologous genes allows for the search of conserved regulatory modules, in particular, via identification of transcription factor binding sites in promoters. Besides this the data on evolutionary conservation of expression profiles will contribute to the translation of knowledge gained through studies of model species to non-model plants. 